\title{
All-photoplastic, soft cantilever cassette probe for scanning force microscopy
}

\author{
G. Genolet, ${ }^{\text {a) }}$ M. Despont, and P. Vettiger \\ Zurich Research Laboratory, IBM Research, CH-8803 Rüschlikon, Switzerland \\ D. Anselmetti \\ Scientific Services, Physics, Novartis Services AG, CH-4002 Basel, Switzerland \\ N. F. de Rooij \\ Institute of Microtechnology, University of Neuchâtel, CH-2000 Neuchâtel, Switzerland
}

(Received 23 August 1999; accepted 3 December 1999)

\begin{abstract}
Multiple single-lever probes for scanning force microscopy arranged in a cassette design and made of a low-stiffness photoplastic material have been developed and successfully tested by imaging DNA molecules. The new concept consists of a column of a one-dimensional array of cantilevers with integrated tips, the first of which is used for imaging and the others are spares in case the first one becomes degraded. When this happens, the lever is mechanically removed from the chip so that the next spare becomes ready for scanning. The probes are fabricated of a photoplastic material, which allows simple batch fabrication and facilitates realization of specific mechanical properties for cantilevers-such as low stiffness - that are very difficult to achieve with classical silicon technology. The batch fabrication process, based on spin coating and subsequent near-ultraviolet exposure and development steps of the photoplastic SU-8, results in well-controlled and uniform mechanical properties of the probes within the same chip, as well as for different chips over a $4 \mathrm{in}$. wafer. Successful images of soft, condensed-matter samples were taken using these cassette probes, making consecutive readdressing of the same DNA molecule with two different cantilevers possible. Thus, a worn-out cantilever can be replaced by a new fresh one with only small positional adjustments and without any changes in the operating conditions. This ease of operation was provided by the cassette concept and the excellent uniformity of the mechanical cantilever characteristics.
\end{abstract}

\section{INTRODUCTION}

In scanning probe microscopy (SPM), single cantilevers are mounted in the microscope and used as long as their tips perform well for the image resolution required. Defective cantilevers are replaced by mounting a new cantilever probe in the apparatus. This change of cantilever requires readjustments of the laser detection scheme and of the operation parameters, which can be rather time consuming. This is even more problematic when operating the cantilever in a nonconventional environment where cantilever replacement is difficult and time consuming. A cassette of cantilevers with several spare probes can greatly reduce the time required to replace and adjust damaged cantilevers. The cassette in conjunction with an appropriate holder allows the damaged cantilever to be broken off and replaced with a new spare with the same mechanical properties from the cassette. Special alignment structures on the holder and cassette ensure that the new cantilever is positioned the same as the one broken off, thus eliminating readjustments. We present the successful fabrication and demonstrate the operation of such a cassette of cantilevers.

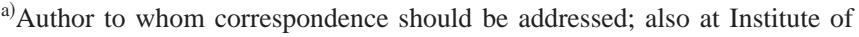
Microtechnology, University of Neuchâtel, Jaquet-Droz 1, CH-2000 Neuchâtel, Switzerland; electronic mail: gen@zurich.ibm.com

\section{CANTILEVER CASSETTE DESIGN}

A one-dimensional (1D) array arrangement of cantilevers [Fig. 1(a)] allows spare cantilevers to be integrated on the same chip as the front cantilever, which is used to scan the sample. Whenever it must be replaced, because the tip or cantilever is damaged or dirty, a stylus is pushed from the top to break off the front lever [Fig. 1(b)], and the next lever is pushed into position. This concept of having spare cantilevers in the same chip allows more than one probe with very similar properties to be integrated on the same chip, making cantilever replacement and adjustment much easier.

Such cantilever cassettes were fabricated using a photoplastic material for the entire chip, that is, for tips, levers, and body of the probe. The photoplastic used to fabricate the soft cantilever cassette is SU-8, an epoxy-based, negativetone, near-ultraviolet (UV) photoresist invented and developed at IBM. ${ }^{1,2}$ This photoplastic is used increasingly in the growing field of microelectromechanical systems (MEMS) as a photoresist as well as structural material. ${ }^{3,4}$ Its small Young's modulus ( $\sim 4 \mathrm{GPa}$, about 40 times smaller than that of silicon) and the freedom of parameter design it allows make SU-8 photoplastic a well-suited material for the fabrication of soft scanning force microscopy (SFM) probes. Such soft photoplastic SFM probes with a small spring constant $(<3 \mathrm{~N} / \mathrm{m})$ consisting of single cantilevers with integrated tips have been fabricated of this material and success- 


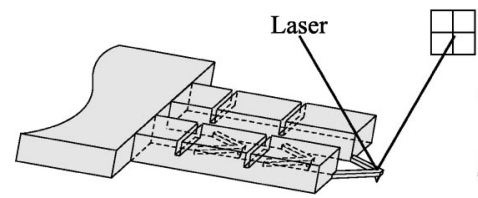

(a)

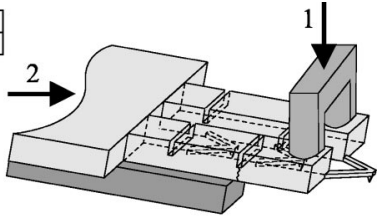

(b)
FIG. 1. Cantilever cassette principle. (a) The first cantilever is used for scanning and spare cantilevers are arranged in a 1D column array behind the first one. (b) A stylus (1) is pushed down to break off the damaged cantilever so that the first spare cantilever can be pushed (2) into the same position as the old one, precisely determined by mechanical alignment structures on cassette and holder.

fully tested. ${ }^{5}$ In addition, the crosslinked photoplastic is chemically inert and temperature-stable up to $200^{\circ} \mathrm{C}$.

\section{FABRICATION PROCESS}

The fabrication process for photoplastic cantilever cassettes is based on spin coating and subsequent near-UV exposure and development of SU-8 photoresist on a prestructured silicon wafer. Levers and integrated tips are hence obtained in a very similar manner as the silicon nitride cantilevers demonstrated by Albrecht et al. ${ }^{6}$

The starting material is a double-side polished $100 \mathrm{~mm}$ $\langle 100\rangle$ silicon wafer. Alignment marks are etched into the backside of the wafer to facilitate alignment of the thick body layers. Pyramidal holes are first etched into the silicon wafer using an anisotropic wet etchant such as $\mathrm{KOH}$ to obtain a mold for the tip [Fig. 2(a)]. Thermal, low-temperature, wet oxidation of the pits is then performed to obtain very
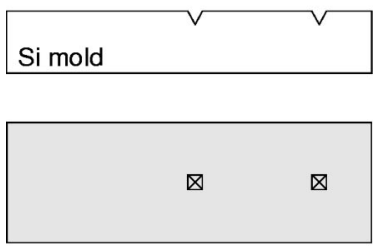

(a)

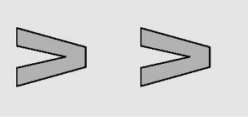

(b)

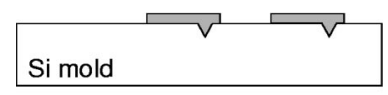

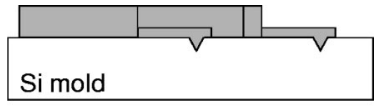

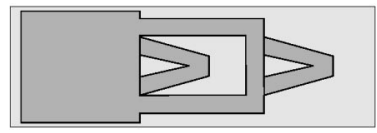

(c)
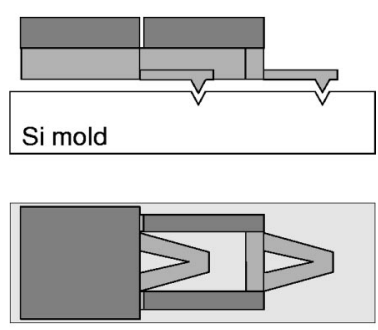

(d)
FIG. 2. Process flow with cross section and top view of the cantilever cassette fabrication. (a) Pyramidal hole formation and oxidation sharpening. (b) Spin coating, exposure and development of SU-8 cantilevers; integrated tips are formed by the resist molded in the pyramidal pit. (c) Spin coating and exposure of the first layer for the body (this layer is shown developed). (d) Spin coating and exposure of the second body layer with trenches to allow tips to be broken off, development of the body layers and unmolding of the entire cassette.

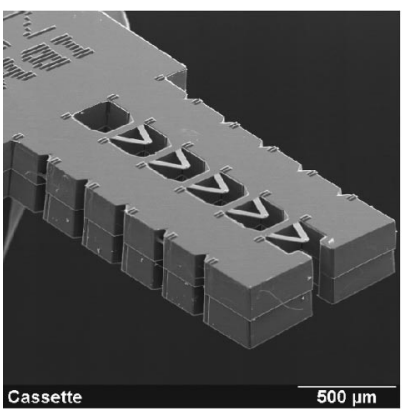

(a)

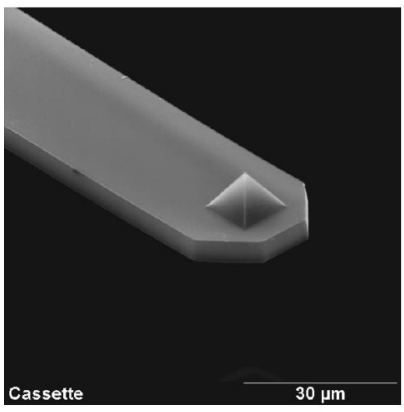

(c)

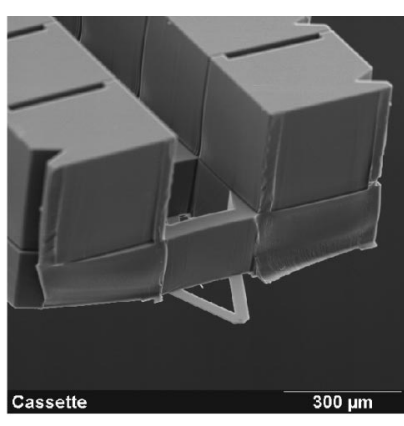

(b)

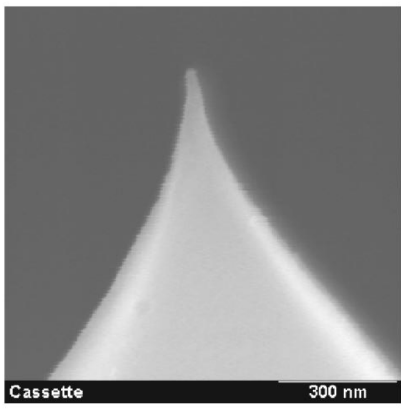

(d)
FIG. 3. (a) SU-8 cantilever cassette probe. The first lever is protected for ease of handling. (b) After breaking off protective blocks, a cantilever is made ready for scanning. Trenches to facilitate the breaking off are clearly visible. (c) Closeup of a cantilever beam with the pyramidal photoplastic molded tip. (d) Closeup of a tip. Radii of curvature smaller than $15 \mathrm{~nm}$ have been measured.

sharp tip molds. Once this silicon mold is ready, the first layer of SU-8 is spin coated onto the wafer, with the tips being defined by the resist that fills up the etched pyramidal pit. A masked exposure with a near-UV light source and subsequent development leave crosslinked photoplastic material that defines the cantilevers with a thickness determined by the thickness of this resist layer [Fig. 2(b)].

A thicker layer of SU-8 is then spin coated on to yield the first body layer. After exposure of this layer, a second body layer is spin coated and exposed. Both layers are then developed simultaneously. The chips are finally mechanically released from the silicon wafer mold without damaging it [Fig. 2(d)], so that after cleaning the mold can be reused for the fabrication of a new batch. This releasing step can be simplified by using a thin sacrificial layer under the SU-8. Finally, $40 \mathrm{~nm}$ of gold is evaporated onto the backside of the levers to achieve sufficient reflection for optical detection. The breaking-off position between the cantilevers is well defined due to trenches defined in the second body layer.

\section{PROBE CHARACTERISTICS}

Figure 3 shows scanning electron micrographs of a fabricated photoplastic SU-8 SFM cantilever cassette with integrated sharp tips. Each cassette is composed of five identical cantilevers arranged in a 1D array. Triangular cantilevers and beam cantilevers have been fabricated. The cantilevers are $190 \mu \mathrm{m}$ long and $40 \mu \mathrm{m}$ wide for the triangle-shaped levers 


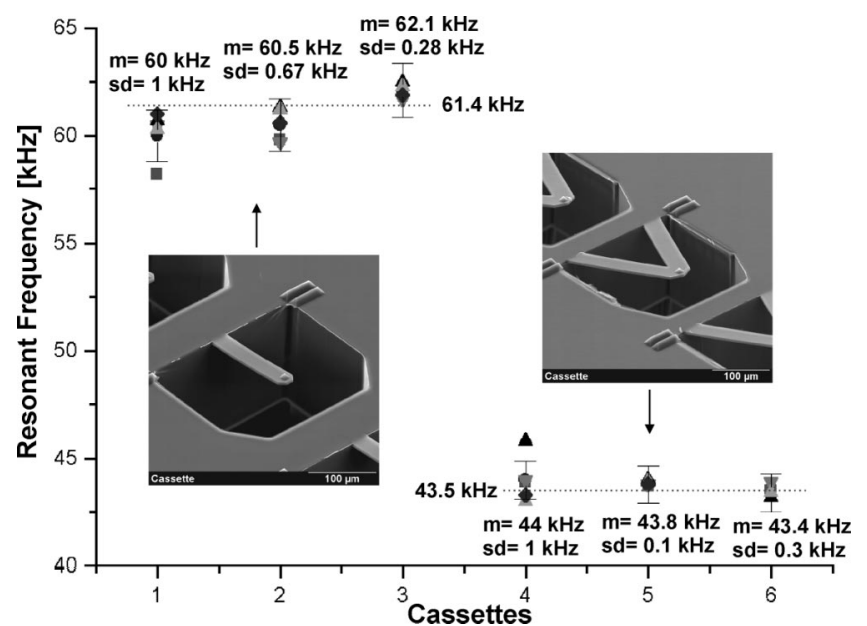

FIG. 4. Homogeneity of the resonant frequency of the cantilevers (beam and triangle shape) within a cassette and among different cassettes. The error bar for each cassette was set to $2 \%$ of the average value of the resonant frequency of the levers of the cassette. The theoretical resonant frequency for the beams and triangle levers is 61.4 and $43.5 \mathrm{kHz}$, respectively. The mean value and standard deviation of the resonant frequency of each of the six cassettes measured are shown.

[Fig. 3(a)] and $160 \mu \mathrm{m}$ long and $30 \mu \mathrm{m}$ wide for the beams. The thickness of both types of cantilevers is around $5.3 \mu \mathrm{m}$. These dimensions yield a theoretical stiffness of 0.86 and 1.1 $\mathrm{N} / \mathrm{m}$, respectively, with corresponding resonant frequencies of 43.5 and $61.4 \mathrm{kHz}$. The measured resonant frequencies differ less than $3 \%$ from the theoretical ones. This difference is mainly due to the measurement accuracy of the lever dimensions and of the resonant frequencies, as well as the approximations of the mechanical properties of the SU-8 material. The close proximity and the well-controlled lever thickness by the spin-coating process yield the same controlled and uniform mechanical characteristics within a cassette. Resonant frequencies of different levers of the same cassette are within $2 \%$ of the average value of the resonant frequency of the cassette (Fig. 4). This homogeneity of the mechanical properties is also very good for cassettes fabricated on a 4 in. silicon wafer mold. This excellent uniformity of mechanical properties within and among cassettes is important for practical use because only slight changes of the cantilever-driving parameters are needed after changing the probes.

Photoplastic tips produced in this manner have been measured to have a radius of curvature of less than $15 \mathrm{~nm}$, determined by scanning electron microscopy (SEM) measurements [Fig. 3(d)]. The tip height is $8.3 \mu \mathrm{m}$.

\section{RESULTS AND DISCUSSION}

Previous experiments with photoplastic single levers ${ }^{5}$ showed a lateral resolution of 5-6 $\mathrm{nm}$ and a vertical resolution of about $0.1 \mathrm{~nm}$. Experiments with single levers in solutions of various $\mathrm{pH}$ showed very good stability of the resonant frequency over time. ${ }^{7}$ The performance of the epoxy cantilever cassettes has also been investigated in atomic force microscopy (AFM) experiments on DNA-plasmid mol-

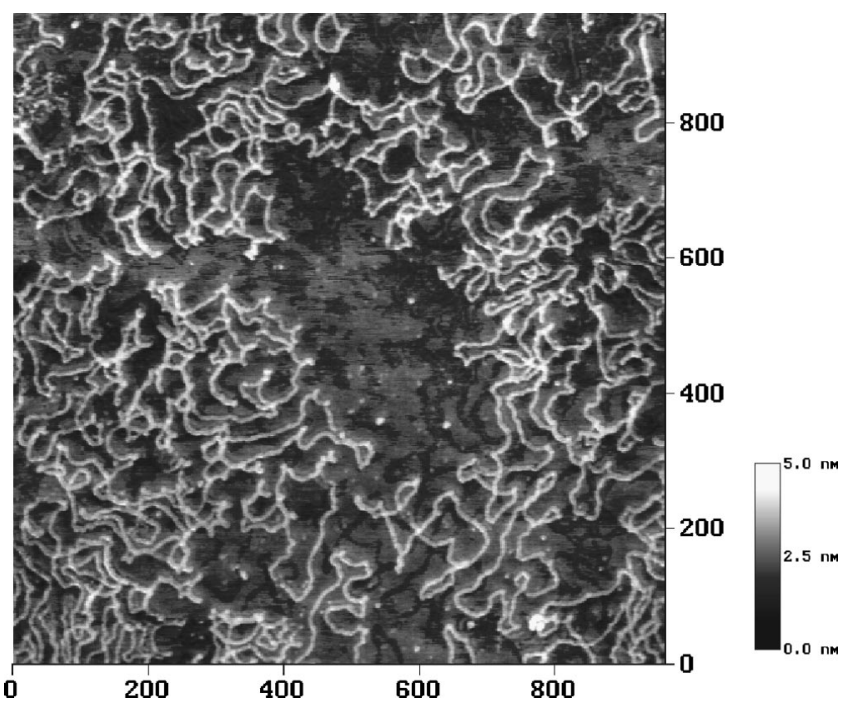

FIG. 5. AFM image $\left(1 \times 1 \mu \mathrm{m}^{2}\right)$ of DNA-plasmid molecules (see the text for details).

ecules in tapping mode under ambient conditions. The cassettes used were composed of cantilever beams. The resonant frequencies of the cantilever cassettes tested were found to be very homogeneously distributed around $61-62 \mathrm{kHz}$. For the tapping mode AFM experiments reported, the driving frequency was typically set $1 \mathrm{kHz}$ below resonance. The homogeneity in resonant frequency from one cantilever to another was so accurate that we were able to run the various experiments at a preset driving frequency of $60.9 \mathrm{kHz}$ for all cantilevers tested.

As a standard experiment, DNA plasmids (pGEM-3Zf vector from Promega Corporation, Madison) immobilized on $\mathrm{NH}_{2}$-functionalized glass were imaged (Fig. 5). Figure 6(a) shows a single DNA molecule imaged with a deteriorated tip, whereas Fig. 6(b) shows the same molecule scanned by the first spare cantilever of a cassette at full resolution. This imaging of the same molecule by two different levers was done with small positional adjustments and without any changes in the operating conditions. This ease of operation

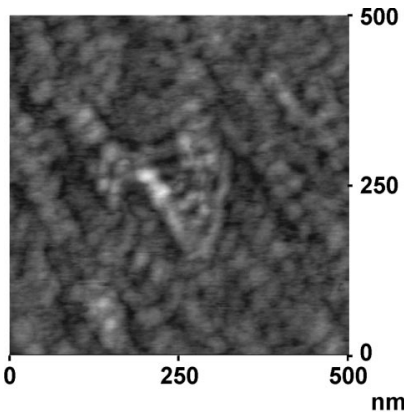

(a)

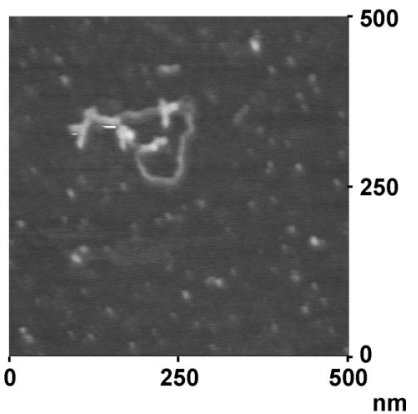

(b)
FIG. 6. DNA-plasmid molecule imaged with different cantilevers. The $z$ scale was color adjusted to $5 \mathrm{~nm}$. (a) Image of the molecule with a deteriorated tip of the first lever of a cassette. The resolution is very poor. (b) Image of the same molecule with the second lever of a cassette with a better tip. 
was provided by the cassette concept and the excellent uniformity of mechanical cantilever characteristics.

\section{CONCLUSIONS}

The fabrication and application of new, soft, photoplastic SFM probes has been demonstrated. Simple molding and replication techniques, inexpensive photoplastic material, reusable molds and several levers per probe make this very attractive for fabricating low-cost photoplastic SFM probes. The very good homogeneity of the cantilevers within a cassette and among cassettes over a 4 in. wafer coupled with an appropriate holder is of great interest for future automated applications.

\section{ACKNOWLEDGMENTS}

The authors thank G. Binnig, U. Drechsler, U. Dürig, W. Häberle, M. Lutwyche, H. Rothuizen, R. Stutz, and R. Widmer of the Micro- and Nanomechanics group at the IBM Zurich Research Laboratory for technical assistance and con- structive discussions, J. Brugger at the MESA Institute of the University of Twente for helpful discussions, and H. Lorenz of Mimotec SA for his valuable knowledge of the SU-8 photoplastic.

${ }^{1}$ N. LaBianca and J. D. Gelorme, in Advances in Resist Technology and Processing XII, edited by R. D. Allen, Proceedings SPIE Vol. 2438 (SPIE, Bellingham, WA, 1994) pp. 846-852.

${ }^{2}$ K. Y. Lee, N. LaBianca, S. A. Rishton, S. Zolgharnain, J. D. Gelorme, J. Shaw, and T. H.-P. Chang, J. Vac. Sci. Technol. B 13, 3012 (1995).

${ }^{3}$ M. Despont, H. Lorenz, N. Fahrni, J. Brugger, P. Renaud, and P. Vettiger, in Proceedings of the IEEE 10th International Workshop on Micro Electro Mechanical Systems. An Investigation of Micro Structures, Sensors, Actuators, Machines and Robots (IEEE, Piscataway, NJ, 1997), pp. $518-$ 522.

${ }^{4}$ H. Lorenz, M. Despont, N. Fahrni, J. Brugger, P. Renaud, and P. Vettiger, Sens. Actuators A 64, 33 (1998).

${ }^{5}$ G. Genolet, J. Brugger, M. Despont, U. Drechsler, P. Vettiger, N. F. de Rooij, and D. Anselmetti, Rev. Sci. Instrum. 70, 2398 (1999).

${ }^{6}$ T. R. Albrecht, S. Akamine, T. E. Carver, and C. F. Quate, J. Vac. Sci. Technol. A 8, 3386 (1990).

${ }^{7} \mathrm{G}$. Genolet et al. (unpublished). 